\title{
Effect of Addition of Lanthanum on the Hydrogen Storage Properties of TiFe Alloy
}

\author{
Md Meraj Alam¹,2 (md.meraj.alam@uqtr.ca), Pratibha Sharma², Jacques Huot ${ }^{1}$ \\ ${ }^{1}$ Université du Québec à Trois-Rivières, Trois-Rivières (Québec) G9A 5H7, Canada \\ ${ }^{2}$ Indian Institute of Technology Bombay, Mumbai 400076, Maharashtra, India
}

TiFe is one of the good candidates for the solid-state hydrogen storage. Despite having fast kinetics and fairly good capacity, it's first hydrogenation is difficult. In this study, we investigated the effect of addition of lanthanum on the hydrogen storage properties of TiFe alloy. As the melting point of lanthanum is much lower than the ones of the other two elements, synthesis by casting was impossible. Instead ball milling was used to synthesize the compound. It was found that the TiFe alloy is formed after 5 hours of milling. The hydrogen storage properties were measured at room temperature and at a pressure of up to 40 bars on a home-made Sievert's apparatus. For the first hydrogenation, the alloy absorbed $1 \mathrm{wt} . \%$ of hydrogen in less than 5 minutes. But, the first de-hydrogenation showed a reduced capacity from $1 \mathrm{wt} \%$ to $0.65 \mathrm{wt} \%$ i.e., a reduction of 0.35 $\mathrm{wt} \%$. Further hydrogenation and dehydrogenation shows no further loss in capacity. To understand the loss in capacity, the X-ray diffraction of fully hydrogenated and fully dehydrogenated samples were performed. But from these diffraction patterns a secondary phase was observed. The presence of this secondary phase may explain the loss of capacity. Possible crystal structure of this phase will be discussed. 